PROCEEDINGS OF THE

AMERICAN MATHEMATICAL SOCIETY

Volume 131, Number 7 , Pages 2089-2096

S 0002-9939(03)06971-5

Article electronically published on February 5, 2003

\title{
A ZERO TOPOLOGICAL ENTROPY MAP WITH RECURRENT POINTS NOT $F_{\sigma}$
}

\author{
PETRA ŠINDELÁŘOVÁ \\ (Communicated by Michael Handel) \\ Dedicated to Professor Jaroslav Smital on the occasion of his 60th birthday
}

\begin{abstract}
We show that there is a continuous map $\chi$ of the unit interval into itself of type $2^{\infty}$ which has a trajectory disjoint from the $\operatorname{set} \operatorname{Rec}(\chi)$ of recurrent points of $\chi$, but contained in the closure of $\operatorname{Rec}(\chi)$. In particular, $\operatorname{Rec}(\chi)$ is not closed. A function $\psi$ of type $2^{\infty}$, with nonclosed set of recurrent points, was found by H. Chu and J. Xiong [Proc. Amer. Math. Soc. 97 (1986), 361-366]. However, there is no trajectory contained in $\overline{\operatorname{Rec}(\psi)} \backslash \operatorname{Rec}(\psi)$, since any point in $\overline{\operatorname{Rec}(\psi)}$ is eventually mapped into $\operatorname{Rec}(\psi)$. Moreover, our construction is simpler.

We use $\chi$ to show that there is a continuous map of the interval of type $2^{\infty}$ for which the set of recurrent points is not an $F_{\sigma}$ set. This example disproves a conjecture of A. N. Sharkovsky et al., from 1989. We also provide another application of $\chi$.
\end{abstract}

\section{INTRODUCTION AND PRELIMINARIES}

The main result of our paper is in Section 3 It states that, for any $f$ in the class $C(I, I)$ of continuous maps of the unit interval, the condition "the set $\operatorname{Rec}(f)$ of recurrent points is an $F_{\sigma}$ set" is strictly stronger than " $f$ is of type less than or equal to $2^{\infty}$ " (cf. Theorems 3.3 and 3.4). Recall that a point $x \in I$ is recurrent if it belongs to its $\omega$-limit set $\omega_{f}(x)$. The map $f$ is of type $2^{n}$ or $2^{\infty}$ if the periods of periodic points of $f$ are the numbers $1,2, \ldots, 2^{n}$, or all powers of 2 , respectively. Recall that $f \in C(I, I)$ has zero topological entropy if and only if it is of type $\leq 2^{\infty}$ (Misiurewicz [10]; cf. also [3]).

Section 2 contains an auxiliary result. Theorem 2.2 exhibits a map of type $2^{\infty}$ with nonclosed set $\operatorname{Rec}(f)$, and with some other properties. We make use of this result in the proof of Theorem 3.3 but it is interesting in itself. Section 4 contains some remarks, an open problem, and another application of Theorem 3.3.

In the sequel, we use the standard terminology and notation as, e.g., in [3]. So we only recall a few items. By $f^{n}$ we denote the $n$th iterate of $f$. The orbit (resp. trajectory) of a set $A$, written $\operatorname{Orb}_{f}(A)\left(\operatorname{resp} . \operatorname{Traj}_{f}(A)\right)$, is the smallest

Received by the editors January 7, 2002

2000 Mathematics Subject Classification. Primary 26A18, 37E05.

Key words and phrases. Topological entropy, recurrent points, periodic points, $\omega$-limit set.

This research was supported, in part, by contracts 201/00/0859 from the Grant Agency of Czech Republic and CEZ:J10/98:192400002 from the Czech Ministry of Education. The support of these institutions is gratefully acknowledged. 
set containing $A$ that is closed under both images and preimages with respect to $f$ (resp. the smallest set containing $A$ closed under $f$ ). A periodic point $p$ of period $n$ is repelling if the one-sided derivatives of $f^{n}$ at $p$ have absolute values greater than 1. By $\operatorname{Per}(f)$ we denote the set of periodic points of $f$, by $\|\cdot\|$ the uniform norm, and by $\bar{A}$ the closure of a set $A$.

\section{NON-CLOSED SET OF RECURRENT POINTS}

We exhibit an auxiliary map $\chi \in C(I, I)$ of type $2^{\infty}$ for which the set of recurrent points is not closed. A map with similar property is given in [5]. However, our construction is simpler and the map has some additional properties which are summarized in Theorem 2.2 below. We use this example in the proof of our main result. But there are other applications; cf. Remark 4.2.

We start with a continuous map $\mu: I \rightarrow I$, which is given by $\mu(0)=1, \mu\left(\frac{2}{5}\right)=\frac{3}{5}$, $\mu\left(\frac{3}{5}\right)=\frac{1}{5}, \mu\left(\frac{4}{5}\right)=\frac{2}{5}$, and $\mu(1)=0$, and which is linear on the intermediate intervals. Its graph is displayed in the next figure.

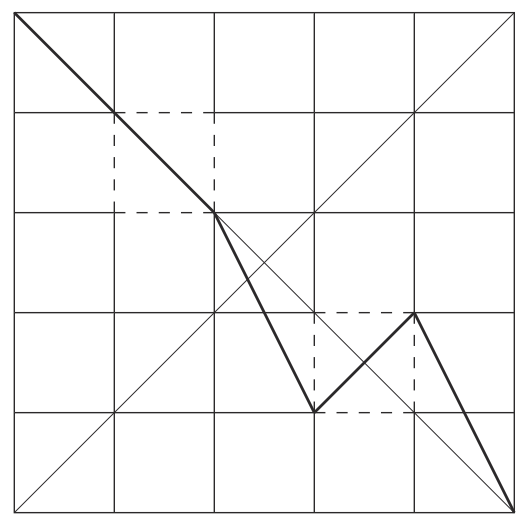

For simplicity, put $I_{1}=\left[\frac{1}{5}, \frac{2}{5}\right]$ and $J_{1}=\left[\frac{3}{5}, \frac{4}{5}\right]$. It is easy to see that $\operatorname{Per}(\mu)=$ $\left\{0,1, \frac{7}{15}\right\} \cup I_{1} \cup J_{1}$. Specifically, $\frac{7}{15}$ is a repelling fixed point, $\{0,1\}$ a repelling periodic trajectory of period 2 , and $\mu^{2}$ is the identity on $I_{1} \cup J_{1}$. Hence, $\mu$ is of type $2^{2}$ and

$$
\text { the isolated points of } \operatorname{Per}(\mu) \text { are repelling. }
$$

Before stating the next proposition recall that a Cantor set $Q \subset I$ is any nonempty, compact set without isolated points containing no intervals. A point $x \in Q$ is extremal if it is isolated in $Q$ from the left or from the right. In other words, extremal points of $Q$ are the end-points of intervals contiguous to $Q$, except for 0 and 1.

Proposition 2.1. There is a continuous map $\chi_{\infty}$ in $C(I, I)$ such that

(i) $\chi_{\infty}$ is of type $2^{\infty}$ with a unique infinite $\omega$-limit set, a Cantor set $Q$;

(ii) $\chi_{\infty}$ is increasing in a neighborhood of any point $x$ in $Q$ different from $a=\min Q$, and $\chi_{\infty}$ is decreasing at $a$;

(iii) $\chi_{\infty}$ is a bijection on the set $A$ of extremal points in $Q$;

(iv) the points in $\operatorname{Per}\left(\chi_{\infty}\right)$ are isolated and repelling;

(v) any point in the orbit of a in $Q$ is in the closure of $\operatorname{Per}\left(\chi_{\infty}\right)$ from each side;

(vi) the $\chi_{\infty}$-preimage of any point $x \in I$ is finite. 
Proof. Let $\alpha, \beta$ be the increasing affine mappings such that $\alpha(I)=I_{1}$ and $\beta(I)=J_{1}$ and, for a positive integer $n$, let $I_{n}=\alpha^{n}(I)$ and $J_{n}=\beta^{n}(I)$. Let $\left\{\chi_{k}\right\}_{k=1}^{\infty}$ be the sequence of maps in $C(I, I)$ such that $\chi_{1}=\mu$, and $\chi_{2}$ is obtained by pasting into the square $I_{1} \times J_{1}$ a five times reduced copy of the graph of $\mu$, i.e.,

$$
\chi_{2}(x)= \begin{cases}\mu(x) & \text { if } x \notin I_{1}, \\ \frac{1}{5} \mu(5 x-1)+\frac{3}{5} & \text { if } x \in I_{1} .\end{cases}
$$

Note that $I_{1}$ is a maximal periodic interval both of $\chi_{1}$ and $\chi_{2}$, of period 2, with $\chi_{1}\left(I_{1}\right)=\chi_{2}\left(I_{1}\right)=J_{1}$. Also, $I_{2}$ is a periodic interval of $\chi_{1}$ and $\chi_{2}$, of period 4 , which is maximal for $\chi_{2}$ but not for $\chi_{1}$. Since $\chi_{1}\left(I_{2}\right)=\chi_{2}\left(I_{2}\right)=J_{2}$, and since the length of $I_{2}$ and $J_{2}$ is $1 / 25$, we can paste into the square $I_{2} \times J_{2}$ the 25-times reduced copy of the graph of $\mu$, to obtain $\chi_{3}$. We proceed inductively. $\chi_{n+1}$ is obtained from $\chi_{n}$ by pasting into the square $I_{n} \times J_{n}$ the $5^{n}$-times reduced copy of the graph of $\mu$.

Consequently,

$$
\operatorname{Per}\left(\chi_{k+1}\right)=\left(\operatorname{Per}\left(\chi_{k}\right) \backslash \operatorname{Traj}_{\chi_{k}} I_{k}\right) \cup \operatorname{Traj}_{\chi_{k}} \alpha^{k}(\operatorname{Per}(\mu)) .
$$

Since $\left\|\chi_{k+1}-\chi_{k}\right\|=\frac{1}{5}|| \chi_{k}-\chi_{k-1} \|$ for $k \geq 1$, the sequence $\left\{\chi_{k}\right\}_{k=1}^{\infty}$ has a uniform limit $\chi_{\infty}$.

Now we can prove the properties (i)-(vi).

(i) Since $I_{k}$ is a maximal periodic interval of $\chi_{k}$ of period $2^{k}$, and the periods of points in $\operatorname{Per}(\mu)$ are at most $4, \chi_{k+1}$ is of type $2^{k+2}$. Block's Stability Theorem [2] implies that $\chi_{\infty}$ is of type $2^{\infty}$. Since, for any $s \geq k, I_{k}$ is a maximal periodic interval of $\chi_{s}$ of period $2^{k}$, the same is also true for $\chi_{\infty}$. Moreover, $\operatorname{Traj}_{\chi_{\infty}}\left(I_{k}\right)$ is the unique trajectory of maximal periodic intervals of $\chi_{\infty}$ of period $2^{k}$, and since its Lebesgue measure is $\left(\frac{2}{5}\right)^{k}, Q=\bigcap_{k=1}^{\infty} \operatorname{Traj}_{\chi_{\infty}}\left(I_{k}\right)$ is a Cantor set (of measure zero). It is well known that then $Q$ is the unique infinite $\omega$-limit set for $\chi_{\infty}$ (cf., e.g., [4]).

(ii) Clearly, $\chi_{k}$ is monotone on any interval from the trajectory of $I_{k}$, and since $\chi_{\infty}(x)=\chi_{k}(x)$ for $x \notin I_{k}, \chi_{\infty}$ is monotone on any interval from the trajectory of $I_{k}$, except for $I_{k}$ itself. To finish the proof note that

$$
\{a\}=\bigcap_{k=1}^{\infty} I_{k} .
$$

(iii) By (ii), $\chi_{\infty}(x) \in A$ if $x \in A$. Hence, $\chi_{\infty}(A) \subset A$. Since $\chi_{\infty}(Q)=Q$, any point $y \in A$ has a preimage $x \in Q$. By (ii), $x$ must be in $A$.

(iv) By (2.3), $\operatorname{Per}\left(\chi_{k}\right) \backslash \operatorname{Orb}_{\chi_{k}}\left(I_{k}\right)$ is a finite set. Thus, the points of $\operatorname{Per}\left(\chi_{\infty}\right)$ are isolated. The rest follows by (2.1).

(v) The end-points $a_{k}<b_{k}$ of $I_{k}$ are in $\operatorname{Per}\left(\chi_{k}\right) \subset \operatorname{Per}\left(\chi_{\infty}\right)$. By (2.4), point $a$ satisfies the condition. By (ii), the same is true for the points $\chi_{\infty}(a)$ and $\chi_{\infty}^{-1}(a) \cap Q$. The result now follows by induction.

(vi) This follows from (2.4) since $\chi_{\infty}$ is piecewise linear on $I \backslash I_{k}$, and is constant on no subinterval of $I$.

Theorem 2.2. There is a map $\chi \in C(I, I)$ of type $2^{\infty}$ such that

(i) $\chi$ has a unique infinite maximal $\omega$-limit set $\tilde{\omega}=R \cup P$, where $R$ is a Cantor set, and $P=\left\{v_{n}\right\}_{n-\infty}^{\infty}$ is an infinite set of points isolated in $\tilde{\omega}$ such that $\chi\left(v_{n}\right)=v_{n+1}$, for any $n$; 
(ii) $P \subset \overline{\operatorname{Per}(\chi)} \backslash \operatorname{Per}(\chi)$;

(iii) the set $\operatorname{Rec}(\chi)$ is not closed;

(iv) any point in $\operatorname{Per}(\chi)$ is isolated in $\omega(\chi)$, and repelling;

(v) $\chi$ is monotone in a neighborhood of any $p \in P$.

Proof. We obtain $\chi$ from the map $\chi_{\infty}$ described in Proposition 2.1 by Denjoy's method of blowing up the orbit of $a=\min Q$ (see [6]; for applications of this method just to maps of type $2^{\infty}$ see [4]). Thus, we replace any point in $\operatorname{Orb}_{\chi_{\infty}}(a)$ by a suitable compact interval, and compress the rest to fit the interval $I$. This is possible by Proposition 2.1 (vi). We obtain a nondecreasing continuous map $\varphi$ from $I$ onto $I$ such that $\chi_{\infty} \circ \varphi=\varphi \circ \chi$. By Proposition 2.1 (iii), $\operatorname{Orb}_{\chi_{\infty}}(a) \cap A$ can be rearranged as $\left\{a_{n}\right\}_{n-\infty}^{\infty}$, where $a_{0}=a$ such that $\chi_{\infty}\left(a_{n}\right)=a_{n+1}$, for any $n$.

Thus, any $a_{n}$ is replaced by a closed wandering interval $A_{n}$ so that $\varphi\left(A_{n}\right)=$ $\chi_{\infty}^{n}(a)=a_{n}$. Let $R$ be the Cantor set such that $\varphi(R)=Q$. Since $\omega_{\chi_{\infty}}(a)=Q$, we have $\omega_{\chi}(x)=R$, for any $x \in A_{n}$. Since the blowing up an orbit preserves the ordering, Proposition 2.1 (v) implies that for any $n$ the end-points $u_{n}$ and $v_{n}$ of $A_{n}$ belong to $\overline{\operatorname{Per}(\chi)}$ and hence, to the set $\omega(\chi)$ of $\omega$-limit points of $\chi$. Just one of the end-points, say point $v_{n}$, is not in $R$. Consequently, $v_{n}$ is an isolated point of a maximal $\omega$-limit set $\tilde{\omega}$ of $\chi$, which is of the form $\tilde{\omega}=R \cup\left\{v_{n}\right\}_{n=-\infty}^{\infty}$ (for well-known facts concerning maximal $\omega$-limit sets of maps of type $2^{\infty}$ see, e.g., [3] or [4]). This proves (i) and (ii).

Since $\tilde{\omega}$ is $\chi$-invariant and any point $v_{n}$ is isolated in $\tilde{\omega}, v_{n} \notin \operatorname{Rec}(\chi)$. But $\overline{\operatorname{Per}(\chi)}=\operatorname{Rec}(\chi)$ (cf., e.g., [3]), hence (ii) implies (iii).

The properties (iv) and (v) follow by Proposition 2.1 (ii) and (v).

\section{The SET OF RECURRENT POINTS NOT OF TYPE $F_{\sigma}$}

Sharkovsky and mathematicians from his school claimed that, for any map $f \in$ $C(I, I)$, the property of "zero topological entropy" is equivalent to "Rec $(f)$ is an $F_{\sigma}$ set" (cf. [11], [12], [8], [7], [14], [13], for example). Unfortunately, this is not the case: we give an example of a map $f \in C(I, I)$ with zero topological entropy with $\operatorname{Rec}(f)$ not an $F_{\sigma}$ set. We also show that the other implication is true.

Proposition 3.1. There is a sequence $\left\{f_{n}\right\}_{n=0}^{\infty}$ of maps in $C(I, I)$ such that, for any $n$,

(i) $f_{n}$ is of type $2^{\infty}$;

(ii) any point in $\operatorname{Per}\left(f_{n}\right)$ is isolated in $\omega\left(f_{n}\right)$, and repelling;

(iii) for any $u \in \operatorname{Per}\left(f_{n}\right)$ there is a $v \in \overline{\operatorname{Per}\left(f_{n+1}\right)} \backslash \operatorname{Rec}\left(f_{n+1}\right)$ such that $|u-v|<$ $1 / 2^{n}$

(iv) $\omega\left(f_{n+1}\right) \supset \omega\left(f_{n}\right)$ and $\operatorname{Per}\left(f_{n+1}\right) \supset \operatorname{Per}\left(f_{n}\right)$;

(v) $f_{n}\left|\omega\left(f_{n}\right)=f_{n+1}\right| \omega\left(f_{n}\right)$;

(vi) $\left\|f_{n}-f_{n+1}\right\|<1 / 2^{n}$.

Proof. Let $f_{0}=\chi$ be as in Theorem 2.2. Assume by induction that $f_{n}$ is given. Let $\alpha$ be the unique fixed point of $f_{0}$ in $I$. The idea is to let $\operatorname{Per}\left(f_{n+1}\right)$ be a disjoint union of small affine copies of $\operatorname{Per}\left(f_{0}\right)$. Any such copy $K$ intersects $\operatorname{Per}\left(f_{n}\right)$ exactly at one point $p$, whose relative position in $K$ is the same as the position of $\alpha$ in $\operatorname{Per}\left(f_{0}\right)$.

By (ii), $\operatorname{Per}\left(f_{n}\right)$ is countable so there is a sequence $\left\{p_{i}\right\}_{i=1}^{\infty}$ in $\operatorname{Per}\left(f_{n}\right)$ that contains exactly one point from any periodic trajectory of a point. By (i), the 
period of $p_{i}$ is $2^{k(i)}$. Again by (ii), for any $i$ there is a compact neighbourhood $U_{i}$ of $p_{i}$ such that $U_{i} \cap \operatorname{Per}\left(f_{n}\right)=\left\{p_{i}\right\}$. Moreover, taking $U_{i}$ sufficiently small, we may assume that $f_{n}^{l}\left(U_{i}\right) \cap f_{n}^{m}\left(U_{j}\right) \neq \emptyset, 0 \leq l, m<2^{k(i)}$ imply $i=j$ and $l=m$. Consequently, for any $i>0$ and any $l$ with $0 \leq l<2^{k(i)}$ there is a compact interval $V_{i}^{l}$ contained in the interior of $f_{n}^{l}\left(U_{i}\right)$ and containing $f_{n}^{l}\left(p_{i}\right)$ with the following two properties: All the intervals $V_{i}^{l}$, for a fixed $i$, have the same length, and the point $f_{n}^{l}\left(p_{i}\right)$ divides $V_{n}^{l}$ in the ratio $\alpha /(1-\alpha)$.

For compact intervals $K, L$, let $\xi_{K, L}$ denote the linear increasing map from $K$ onto $L$, and define the map $f_{n+1}: I \rightarrow I$ as follows:

$$
\begin{aligned}
f_{n+1}(x) & =f_{n}(x) \text { if } x \notin f_{n}^{l}\left(U_{i}\right), \\
f_{n+1} \mid V_{i}^{0} & =\xi_{I, V_{i}^{1}} \circ f_{0} \circ \xi_{V_{i}^{0}, I}, \\
f_{n+1} \mid V_{i}^{l} & =\xi_{V_{i}^{l}, V_{i}^{l+1}},
\end{aligned}
$$

for any $i$ and any $l=1, \ldots, 2^{k(i)}-1$, letting $V_{i}^{2^{k(i)}}=V_{i}^{0}$. Then extend $f_{n+1}$ linearly onto the whole of $I$.

To finish the proof it suffices to verify that $f_{n+1}$ satisfies conditions (i)-(vi), with $n$ replaced by $n+1$. Conditions (iv) and (v) are obvious, and (ii) follows by Theorem 2.2 (iv). For a continuous map $\varphi$ of the interval,

$$
\operatorname{Per}(\varphi) \subset \operatorname{Rec}(\varphi) \subset \overline{\operatorname{Per}(\varphi)}=\overline{\operatorname{Rec}(\varphi)} \subset \omega(\varphi) ;
$$

cf., e.g., 3]. Consequently, by Theorem 2.2 (ii), $D=\overline{\operatorname{Per}(\chi)} \backslash \operatorname{Rec}(\chi) \neq \emptyset$. To prove (iii) note that, locally at $u$, the set $\overline{\operatorname{Per}\left(f_{n+1}\right)} \backslash \operatorname{Rec}\left(f_{n+1}\right)$ is an affine copy of $D$. Hence to meet condition (iii) it suffices to take the intervals $U_{i}$ sufficiently small, of diameter less than $2^{-n}$. This will also imply (vi).

Thus, it remains to verify condition (i). We have to show that $f_{n+1}$ has no periodic point of period $\neq 2^{m}$. Assume the contrary: let $p$ be a periodic point of $f_{n+1}$ of period $k \neq 2^{m}$. The trajectory of $p$ must be disjoint from $V=\bigcup_{i=0}^{\infty} \bigcup_{l=0}^{2^{k(i)}-1} f_{n}^{l}\left(V_{i}^{0}\right)$ since $V$ is an invariant set and, by (3.2) and (3.3), $f_{n+1} \mid V$ has zero topological entropy. On the other hand, by (3.1), the trajectories of $p$ cannot be contained in $I \backslash \bigcup_{i=0}^{\infty} \bigcup_{l=0}^{2^{k(i)}-1} f_{n}^{l}\left(U_{i}\right)$. Hence, we may assume that $p \in U_{i} \backslash V_{i}$. Since by (ii) the periodic point $p_{i}$ of $f_{n}$ is repelling, by taking $V_{i}$ sufficiently small we get the same for $p_{i}$ as a periodic point of $f_{n+1}$. This means that the trajectory of $p$ is eventually in the complement of $U_{i}$, and this is a contradiction. Thus, $f_{n+1}$ satisfies condition (i).

The following result is known but we are not able to give a reference. Recall, that $X$ is nonwandering if, for any open set $G \neq \emptyset$ in $X$ there is an integer $n>1$ such that $\varphi^{n}(G) \cap G \neq \emptyset$.

Lemma 3.2. Let $(X, d)$ be a compact metric space, and $\varphi \in C(X, X)$. If $X$ is nonwandering, then $\operatorname{Rec}(\varphi)$ is a $G_{\delta}$ set.

Proof. Our argument is a modification of the proof of Theorem 1.27 in [9]. Let $d$ denote the metric on $X$, and define

$$
F(x)=\inf _{n \geq 1} d\left(x, \varphi^{n} x\right) .
$$

Because of the well-known fact that the set of points of continuity of any function $F$ with domain and range in metric spaces is a $G_{\delta}$ set, it suffices to show that $\operatorname{Rec}(\varphi)$ is the set of points of continuity of $F$. 
First suppose that $x_{0}$ is a point of continuity of $F$. If $F\left(x_{0}\right)=0$, then $x_{0}$ is a recurrent point. If $F\left(x_{0}\right)>0$, then, for some $a>0$ and some neighbourhood $V$ of $x_{0}, F(x)>a$ throughout this neighbourhood. We may assume that $\operatorname{diam}(V)<a$. Since $V$ is not a wandering set, for some $n, \varphi^{n} V \cap V \neq \emptyset$ or, for some $x \in V$, $\varphi^{n} x \in V$. But then $F(x)<a$, which is a contradiction.

To finish the proof, let $x_{0} \in \operatorname{Rec}(\varphi)$. Then for any $\varepsilon>0$ there is an integer $n_{0} \geq 1$ satisfying $d\left(x_{0}, \varphi^{n_{0}} x_{0}\right)<\varepsilon$. Since $\varphi^{n_{0}}$ is continuous, there is a $\delta>0$ such that $d\left(\varphi^{n_{0}} x_{0}, \varphi^{n_{0}} x\right)<\varepsilon$ whenever $d\left(x, x_{0}\right)<\delta$. Hence, for any $x$ with $d\left(x, x_{0}\right)<$ $\min \{\delta, \varepsilon\}$,

$$
d\left(x, \varphi^{n_{0}} x\right) \leq d\left(x, x_{0}\right)+d\left(x_{0}, \varphi^{n_{0}} x_{0}\right)+d\left(\varphi^{n_{0}} x_{0}, \varphi^{n_{0}} x\right)<3 \varepsilon .
$$

Consequently,

$$
\begin{gathered}
F(x)=\inf _{n \geq 1} d\left(x, \varphi^{n} x\right) \leq d\left(x, \varphi^{n_{0}} x\right)<3 \varepsilon, \\
F\left(x_{0}\right)=\inf _{n \geq 1} d\left(x_{0}, \varphi^{n} x_{0}\right) \leq d\left(x_{0}, \varphi^{n_{0}} x_{0}\right)<\varepsilon,
\end{gathered}
$$

and finally, $\left|F(x)-F\left(x_{0}\right)\right|<3 \varepsilon$, i.e., $F$ is continuous in $x_{0}$.

Theorem 3.3. There is a map $f \in C(I, I)$ with zero topological entropy such that $\operatorname{Rec}(f)$ is not an $F_{\sigma}$ set.

Proof. Let $f_{n}$ be as in Proposition 3.1 By (vi), put $f=\lim _{n \rightarrow \infty} f_{n}$. Then by (i) and (vi), $f$ is of type $\geq 2^{\infty}$, and by the Block's Stability Theorem [2] $f$ is of type $\leq 2^{\infty}$. Thus, $f$ is of type $2^{\infty}$. Moreover,

$$
\operatorname{Rec}(f) \text { and } \overline{\operatorname{Per}(f)} \backslash \operatorname{Rec}(f) \text { are dense in } \overline{\operatorname{Per}(f)} .
$$

Indeed, $\operatorname{Rec}(f)$ is dense in $\overline{\operatorname{Per}(f)}$ by (3.4). The other set is dense since by (iii), (iv) and (v), $\bigcup_{n=1}^{\infty} \overline{\operatorname{Per}\left(f_{n}\right)} \backslash \operatorname{Rec}\left(f_{n}\right)$ is dense in $\overline{\operatorname{Per}(f)}$ and, by (3.4) and (v), $\overline{\operatorname{Per}\left(f_{n}\right)} \backslash \operatorname{Rec}\left(f_{n}\right)=\overline{\operatorname{Per}\left(f_{n}\right)} \backslash \operatorname{Rec}(f)$. By Lemma 3.2, $\overline{\operatorname{Per}(f)} \backslash \operatorname{Rec}(f)$ is an $F_{\sigma}$ set. But then $\operatorname{Rec}(f)$ cannot be $F_{\sigma}$ since otherwise, by (3.5), $\overline{\operatorname{Per}(f)}$ would be a countable union of nowhere dense closed sets and hence, of the first category, contrary to the Baire theorem.

Theorem 3.4. Let $f \in C(I, I)$. If $\operatorname{Rec}(f)$ is an $F_{\sigma}$ set, then $f$ has zero topological entropy or, equivalently, is of type $\leq 2^{\infty}$.

Proof. Let $\Sigma$ be the set of infinite sequences $\alpha=\left(a_{1}, a_{2}, \ldots\right)$ with $a_{k}=0$ or 1 , and let $\sigma: \Sigma \rightarrow \Sigma$ be the shift operator $\left(a_{1}, a_{2}, \ldots\right) \mapsto\left(a_{2}, a_{3}, \ldots\right)$. Then $\operatorname{Rec}(\sigma)$ is not an $F_{\sigma}$ set. Indeed, by Lemma 3.2. $\operatorname{Rec}(\sigma)$ is a $G_{\delta}$ set, hence its complement $Y=\Sigma \backslash \operatorname{Rec}(\sigma)$ is an $F_{\sigma}$ set. It is easy to see that both $\operatorname{Rec}(\sigma)$ and $Y$ are dense in $X$. Hence, if $\operatorname{Rec}(\sigma)$ is $F_{\sigma}$, then $X$ would be the union of a countable family of nowhere dense closed sets and hence, of the first category, contrary to the Baire theorem.

Now assume that $f \in C(I, I)$ has positive topological entropy. Then, by [1] (cf. also [3]), there is an integer $n \geq 1$, and a compact set $X \subset I$ such that $f^{n}(X)=X$, and $f^{n} \mid X$ is semiconjugate to $\sigma$, i.e., there is a continuous surjective map $h: X \rightarrow \Sigma$ which is one-to-one with countably many exceptions where the map is two-to-one, and

$$
h \circ f^{n}(x)=\sigma \circ h(x), x \in X
$$


Moreover, for any $y \in \Sigma$,

$$
h^{-1}(y)=\left\{z_{0}, z_{1}\right\} \text { and } z_{0}<z_{1} \text { imply } X \cap\left(z_{0}, z_{1}\right)=\emptyset .
$$

Denote $g=f^{n} \mid X$. By Lemma 2.1 in [1],

$$
\omega_{\sigma}(h(x))=h\left(\omega_{g}(x)\right) \text { for any } x \in X \text {. }
$$

We prove that

$$
h(\operatorname{Rec}(g))=\operatorname{Rec}(\sigma) .
$$

If $x \in \operatorname{Rec}(g)$, i.e., if $x \in \omega_{g}(x)$, then $h(x) \in h\left(\omega_{g}(x)\right)$; hence, by (3.8), $h(x) \in$ $\omega_{\sigma}(h(x))$, i.e., $h(x) \in \operatorname{Rec}(\sigma)$. This shows that $h(\operatorname{Rec}(g)) \subset \operatorname{Rec}(\sigma)$. To prove the converse inclusion, assume that $y \in \operatorname{Rec}(\sigma)$, and let $h^{-1}(y)=\left\{z_{0}, z_{1}\right\}$, possibly with $z_{0}=z_{1}$. If $z_{0}=z_{1}$, then $z_{0} \notin \omega_{g}\left(z_{0}\right)$ would imply $h\left(z_{0}\right) \notin h\left(\omega_{g}\left(z_{0}\right)\right)$; hence, by (3.8),$y \notin \operatorname{Rec}(\sigma)$. This contradiction shows that $y \in h(\operatorname{Rec}(g))$. If $z_{0}<z_{1}$ and $z_{i} \notin \omega_{g}\left(z_{j}\right)$, for any $i, j \in\{0,1\}$, then, by (3.7) $),\left(\omega_{g}\left(z_{0}\right) \cup \omega_{g}\left(z_{1}\right)\right) \cap\left\{z_{0}, z_{1}\right\}=\emptyset$ and again, since $h$ is at most two-to-one, $y \notin \operatorname{Rec}(\sigma)$ which is impossible. Thus, we have $z_{i} \in \omega_{g}\left(z_{j}\right)$, for some $i, j$. But then, by (3.7), $z_{i} \in \omega_{g}\left(z_{i}\right)$, for some $i$, and again $y \in h(\operatorname{Rec}(g))$. This proves 3.9 .

Now to finish the proof of Theorem 3.4 assume that $\operatorname{Rec}(f)$ is an $F_{\sigma}$ set. Since, for any continuous map $\varphi$ of a compact metric space, and any positive integer $m$, $\operatorname{Rec}(\varphi)=\operatorname{Rec}\left(\varphi^{m}\right)$ (cf., e.g., 3, Lemma 4.25), we conclude that $\operatorname{Rec}\left(f^{n}\right)$, and hence, $\operatorname{Rec}(g)=\operatorname{Rec}\left(f^{n}\right) \mid X$, is an $F_{\sigma}$ set. But then, by (3.9), $\operatorname{Rec}(\sigma)$ would be an $F_{\sigma}$ set, a contradiction.

\section{Concluding REMARKS}

Remark 4.1. In fact, Sharkovsky et al., among others, stated that the next three conditions are equivalent: $\left(P_{1}\right) f$ has zero topological entropy; $\left(P_{2}\right)$ the set of periodic points of $f$ is a $G_{\delta}$ set; $\left(P_{3}\right)$ the set of recurrent points of $f$ is an $F_{\sigma}$ set. Unfortunately, this is not true: no two of these conditions are equivalent.

Recently [15] we proved that $\left(P_{2}\right)$ implies $\left(P_{1}\right)$ and we exhibited a map satisfying $\left(P_{1}\right)$, but not $\left(P_{2}\right)$. This map, however, has the property $\left(P_{3}\right)$. By the present paper, Theorems 3.3 and 3.4, $\left(P_{3}\right)$ implies but is not implied by $\left(P_{1}\right)$. There is still an open problem if $\left(P_{2}\right)$ implies $\left(P_{3}\right)$. Our conjecture is that the answer is positive. Under this hypothesis, we get the following ordering: $\left(P_{2}\right)$ is stronger than $\left(P_{3}\right)$, and $\left(P_{3}\right)$ is stronger than $\left(P_{1}\right)$.

Remark 4.2. Let $G$ be a (continuous) triangular map of the square $I^{2}$ defined by $(x, y) \mapsto\left(\chi(x), g_{x}(y)\right)$ where $\chi$ with $\omega$-limit set $\tilde{\omega}=R \cup P$ is the map described in Theorem 2.2, and $g_{x}$ is continuous and nondecreasing for any $x$. Let $g_{x}$ be the identity for any $x \in R$. It is possible to specify the maps $g_{x}$ so that $\operatorname{Per}(\chi) \times I=$ $\operatorname{Per}(G)$ and, for some $a \in P$, the points $u=(a, 0)$ and $v=(a, 1)$ satisfy conditions

$$
\liminf _{n \rightarrow \infty}\left|G^{n}(u)-G^{n}(v)\right|=0, \limsup _{n \rightarrow \infty}\left|G^{n}(u)-G^{n}(v)\right|=1 .
$$

Thus, $G$ restricted to $P \times I$ is chaotic in the sense of $\mathrm{Li}$ and Yorke. On the other hand, the points $u, v$ are not recurrent (since no point in $P$ is), but both belong to the closure of $\operatorname{Per}(G)$, hence, to the center $C(G):=\overline{\operatorname{Rec}(G)}=\omega(\chi) \times I$ of $G$. Consequently, $G$ is chaotic on the center of $G$. Now $G$ can be extended to the whole of $I^{2}$ so that $\{a\} \times I$ intersects $\omega(G)$ exactly at one point. Consequently, $G$ is not 
chaotic on $\omega(G)$. Such a map $G$ cannot be obtained when $\chi$ is replaced by the map from [5] since there the trajectory of any point $a$ in $P$ is eventually in $R$.

\section{REFERENCES}

[1] L. Alsedà, M. Chas and J. Smítal, On the structure of the $\omega$-limit sets for continuous maps of the interval, Discrete dynamical systems, Internat. J. Bifur. Chaos Appl. Sci. Engrg. 9 (1999), no. 9, 1719-1729. MR 2000i:37047

[2] L. Block, Stability of periodic orbits in the theorem of Šarkovskii, Proc. Amer. Math. Soc. 81 (1981), 333-336. MR 82b:58071

[3] L. S. Block and W. A. Coppel, Dynamics in One Dimension, Lecture Notes in Math. 1513, Springer, Berlin - Heidelberg - New York, 1992. MR 93g:58091

[4] A. M. Bruckner and J. Smítal, A characterization of $\omega$-limit sets of maps of the interval with zero topological entropy, Ergod. Th. \& Dynam. Sys. 13 (1993), 7-9. MR 94k:26006

[5] H. Chu and J. Xiong, A counterexample in dynamical systems of the interval, Proc. Amer. Math. Soc. 97 (1986), 361-366. MR 87i:58140

[6] A. Denjoy, Sur les courbes définies par les équations differentielles à la surface du tore, J. Math. Pures Appl. 11 (1932), 333-375.

[7] V. V. Fedorenko, Classification of simple one-dimensional dynamical systems, (Russian) Akad. Nauk Ukrain. SSR Inst. Mat. Preprint 1991, no. 5, 30 pp. MR 92m:58037

[8] V. V. Fedorenko, A. N. Sharkovsky and J. Smítal, Characterization of weakly chaotic maps of the interval, Proc. Amer. Math. Soc. 110 (1990), 141-148. MR 91a:58148

[9] H. Furstenberg, Recurrence in ergodic theory and combinatorial number theory, Princeton University Press, Princeton, 1981. MR 82j:28010

[10] M. Misiurewicz, Horseshoes for mappings of the interval, Bull. Acad. Polon. Sci. Sér. Sci. Math. 27 (1979), 167-169. MR 81b:58033

[11] A. N. Sharkovsky, Classification of one-dimensional dynamical systems, European Conference on Iteration Theory (Caldes de Malavella, 1987), 42-55, World Sci. Publishing, Teaneck, NJ, 1989. MR 92a:58075

[12] A. N. Sharkovsky, S. F. Kolyada, A. G. Sivak and V. V. Fedorenko, Dynamics of Onedimensional Mappings, Naukova Dumka, Kijev, 1989, 216 pp. (Russian) MR 91k:58065

[13] A. N. Sharkovsky, S. F. Kolyada, A. G. Sivak and V. V. Fedorenko, Dynamics of Onedimensional Maps, Kluwer Academic Publishers Group, Dordrecht, 1997. MR 98k:58083

[14] A. N. Sharkovsky, Yu. L. Maistrenko and E. Yu. Romanenko, Difference equations and their applications, Kluwer Academic Publishers Group, Dordrecht, 1993. MR 95j:39001

[15] P. Šindelářová, A zero topological entropy map for which periodic points are not a $G_{\delta}$ set, Ergod. Th. \& Dynam. Sys. 22 (2002), 947-949.

Mathematical Institute, Silesian University in Opava, Bezručovo nám. 13, 74601 Opava, Czech Republic

E-mail address: Petra.Sindelarova@math.slu.cz 